Saudi Journal of Biomedical Research

Abbreviated Key Title: Saudi J Biomed Res ISSN 2518-3214 (Print) |ISSN 2518-3222 (Online)

\title{
Alteration of Coagulation Parameters in Plasmodium Falciparum Malaria Patients and its Correlation with Degree of Parasitaemia in Khartoum state Sudan; 2019
}

\author{
Rawan Abdullah Ibrahim Abdullah ${ }^{1 *}$, Mosab Nouraldein Mohammed Hamad ${ }^{2,3}$, Sahar Eldirderi Gafar Osman ${ }^{2}$, Tamer \\ Imad AldeenTaha Mahmoud ${ }^{1}$, Ehab Mohammed Elmadenah Mohammed Ahmed ${ }^{2,4}$ \\ ${ }^{1}$ Hematology department, Faculty of Medical Laboratory Science, University of Medical Sciences and Technology Sudan \\ ${ }^{2}$ Medical Laboratory Department, Alfajr College for Sciences and Technology Sudan \\ ${ }^{3}$ Medical Laboratory Science Department, Elsheikh Abdallah Elbadri University \\ ${ }^{4}$ Faculty of Medical Laboratory Sciences, Dongola University Sudan
}

\begin{abstract}
DOI: $10.36348 /$ sjbr.2019.v04i12.004 $\quad$ | Received: 12.12 .2019 | Accepted: 24.12 .2019 | Published: 28.12 .2019
*Corresponding author: Rawan Abdullah Ibrahim Abdullah
\end{abstract}

\section{Abstract}

Background: The hemostatic alterations play an important role in malaria pathogenesis and in the progression of disease. In severe infection the accelerated coagulation cascade activity with accelerated fibrinogen turnover increase the bleeding tendency and may lead to DIC. Objectives: This study was aimed to determine the Alteration of coagulation parameters in Plasmodium Falciparum malaria Patients and its correlation with degree of parasitemia in Khartoum state. Sudan; 2019. Material and method: Analytical cross sectional study, 170 blood samples were enrolled in this study. 85 samples of blood were collected from patients with falciparum malaria and 85 samples were collected from healthy individual as control, then PT and APTT will be measured by coagulometer. Result: The mean of PT was significantly high in case than in control $(14.201$ second vs 13.668 second, $(\mathrm{P}$ value $(<0.01)$ ). While there was no differences in the mean of APTT between case and control (33.075 vs 33.192 second ) P value (0.735). Conclusions: Our study concluded that the mean of PT was significantly higher in case than control, While there was no differences in the mean of APTT between case and control, and There was no differences between the PT and APTT of case and control according to gender. And there is no correlation between degree of parasitemia in PT, APTT and age.

Keywords: Malaria, P. falciparum, parasite density, coagulation profile.

Copyright @ 2019: This is an open-access article distributed under the terms of the Creative Commons Attribution license which permits unrestricted use, distribution, and reproduction in any medium for non-commercial use (NonCommercial, or CC-BY-NC) provided the original author and source are credited.

\section{INTRODUCTION}

Malaria is a protozoan disease transmitted by the bite of infected Anopheles mosquito. Despite enormous control efforts, increase in the drug resistance of the parasite, the insecticide resistance of its vectors, human travel and migration have contributed to its resurgence and is a leading cause of mortality and morbidity in developing areas of the world. It is endemic in at least 87 countries, placing approximately 2.5 billion people at risk. Malaria is caused by Plasmodium sp. transmitted to the host by bite of the blood-feeding Anopheles sp. mosquitoes, which inject sporozoite-infected saliva, There are four different types of Plasmodia (P. falciparum, P. vivax, P. ovale, $P$. malariae) that infect humans but $P$. falciparum causes almost all death[1]. Common clinical presentation of infection of severe malaria are severe anemia, acidosis, jaundice, acute renal failure, acute respiratory distress syndrome, hyperparasitemia, shock, and hypoglycemia, disseminated intravascular coagulation, convulsions, hemoglobinuria, impaired consciousness and extreme weakness. These occur mostly with Plasmodium falciparum malaria [2].

The term malaria comes from two medieval Italian words "mal" and "aria," which when put together mean "bad air." This came about before people understood that the disease was passed from a parasite carried by misquotes who lived in the wet ground, and not through "the bad air of swamps.

Malarial infection begins when a person is bitten by an infected female anopheles mosquito and Plasmodium spp (species) parasites in the form of sporozoites are injected into the bloodstream. The sporozoites travel to the liver, multiplying asexually over the next 7-10 days. During this time there are no symptoms. The parasites, now in the form of 
merozoites, emerge from the liver cells in vesicles and travel through the heart to the capillaries of the lungs. The vesicles eventually disintegrate, releasing the merozoites to enter the bloodstream where they invade and multiply in erythrocytes. When the cells burst, the parasites invade more erythrocytes. Clinical symptoms, including fever, occur in synchrony with the rupture of infected erythrocytes and the release of erythrocyte and parasite debris, including malarial pigment (hemozoin) and glycophosphatidylinositol, the putative 'malaria toxin[3,4]'. In some infected blood cells, instead of replicating asexually, the merozoites develop into sexual forms (gametocytes), which circulate in the bloodstream and are ingested during mosquito bites. The ingested gametocytes develop in the mosquito into mature sex cells (gametes) which develop into ookinetes that actively burrow through the mid-gut wall of the mosquito and form oocysts, in which develop thousands of active sporozoites. The oocyst eventually bursts, releasing sporozoites that travel to the salivary glands of the mosquito. The cycle of human infection begins again when the mosquito bites another person [5].

Malaria is usually associated with reduced blood cell counts \& mild to moderate thrombocytopenia is a common association of malaria. The cause of thrombocytopenia is poorly understood, but the immune-mediated lysis, sequestration in the spleen and dyspoietic processes in the marrow with diminished platelet production have all been postulated [6].

\section{Justification}

Malaria is a major health problem in the world, and causes the morbidity and mortality; severe and complicated malaria is caused by plasmodium falciparum. Severe malaria is characterized by a set of clinical and laboratory parameters that are associated with an increased risk of death.

The hemostatic alterations play an important role in malaria pathogenesis and in the progression of disease. In severe infection the accelerated coagulation cascade activity with accelerated fibrinogen turnover increase the bleeding tendency and may lead to DIC. The present study was aimed to determine the effect of malaria parasites on Prothrombin Time and Activated Partial Thromboplastin Time Levels and it's correlation with degree of parasitaemia.

\section{OBJECTIVES}

\section{General objectives}

To determine alteration of coagulation parameters in Plasmodium falciparum malaria Patients and its correlation with degree of parasitaemia in Khartoum state Sudan; 2019

\section{Specific objectives}

- To determine relationship between intensity of parasite and PT level in Sudanese patients.
- To determine relationship between intensity of parasite and APTT level in Sudanese patients.

- To Compare in PT level between case and control group.

- To Compare in APTT level between case and control group.

\section{MATERIAL AND METHODS Study design}

Hospital based case control study

\section{Study duration}

The study was conducted during the period (May 2019- August 2019).

\section{Study setting}

The study was carried out at Almadina Almonawra Center in Khartoum state, Sudan.

Sample size

One hundred and seventy blood samples were collected and distribute as:

- 85 blood sample from malaria patient.

- 85 blood samples from healthy adult.

\section{STATISTICAL ANALYSIS}

Data analysed by statistical package for social science (SPSS).

\section{Ethical consideration}

It was taken from Ministry of Health and then informed consent obtained from each participant.

\section{METHOD}

\section{Specimen collection}

Venous blood collected using sterile disposables plastic syringe after cleaning the vein puncture area with $70 \%$ ethanol, the blood added to anticoagulant ration of $2.5 \mathrm{ml}$ of blood to $0.5 \mathrm{ml}$ citrate (3.2\%) buffer sodium citrate and gently mixed, then PT and APTT measured by Coagulometer. The intensity of parasitaemia was calculated from thick blood film.

\section{Specimen preparation of platelet poor plasma} PPP was prepared by centrifugation of the citrated sample at $1500 \mathrm{rpm}$ for 15 minutes then the plasma transfused in to plane container.

\section{Prothrombin time (PT)}

\section{Principle}

The prothrombin test measures the clotting time of the plasma in the presence of an optimal concentration of tissue extraction (thromboplastin) and indicates the overall efficiency of the extrinsic clotting system. 


\section{Procedure}

PT Liquid thromboplastin brought to $37 \mathrm{c}$ and was well mixed, Coagulometer was adjusted at $37 \mathrm{c}$ and cuvette with magnetic stirrers inside were kept in holes at $37 \mathrm{c}$ for 2 minutes then $200 \mu \mathrm{L}$ of prothrombin liquid thromboplastin added to $100 \mu \mathrm{L}$ of plasma and simultaneously was measured at the end point. Start the stop watch to observe clots.

\section{Normal ranges}

The normal range of PT is between 11-16 seconds.

\section{Activated partial thromboplastin time (APTT)}

\section{Principle}

The test measures the clotting tie of plasma often the activation of contact factors but without added tissue thromboplastin, and so indicates the overall efficiency of intrinsic pathway. To standardized the activation of contact factors. The plasma is first preincubated with kaolin. Standardized phospholipids' is provided to allow the test to be performed on PPP. The test depends at only the contact factor and factor VII and IX, but also on the reactions with factor X, V, II it is also sensitive to the presence of circulating anticoagulants ( inhibitors ) and heparin.

\section{Procedure}

Activated partial thromboplastin reagent was brought to 37c and was well mixed; Coagulometer was adjusted at 37c and cuvette with magnetic stirrer inside was kept in holes at 37c. $100 \mu \mathrm{L}$ of plasma placed in cuvette, $100 \mu \mathrm{L}$ of APTT reagent which was prewarmed at $37 \mathrm{c}$ was added to plasma, mixed well and incubate for 3 minutes, $100 \mu \mathrm{L}$ of $\mathrm{Cacl} 2$ which was prewormed at 37c was added and simultaneously the display the clot when the start of stop watch.

\section{Normal range} seconds

The normal range is typically within 26-40

\section{RESULT}

Hospital based Case control study, 170 blood samples were enrolled in this study.85 samples of blood were collected from patients with malaria and 85 samples were collected from healthy individual as control.
The frequency and percentage were calculated in case whom suffering from malaria according to gender $30(35.3 \%)$ male and $55(64.7 \%)$ female (Table: $1)$.

(Table: 2), frequency and percentage in case according to degree of parasitaemia, one cross (+) was $65(76.5 \%)$ and two cross (++) was $20(23.5 \%)$. The mean of case according to PT was found to be 14.201 second and 13.668 second in control with highly significant $\mathrm{P}$ value (0.00). While the mean of case according to APTT was found to be 33.192 second and in control was found 33.075 second with insignificant $P$ value $(0.735)$ (Table: 3$)$.

The comparison between case and control in PT and APTT according to gender. The mean of PT in male was found to be 14.487 second and female 14.045 second with insignificant $P$ value was found 0.061. While The mean of APTT in male was found to be 33.473second and female 33.038second with insignificant $P$ value was found 0.523.(Table:4)

Table-1: showed frequency and percent between the genders

\begin{tabular}{|l|l|l|}
\hline Gender & Frequency & Percent \\
\hline Male & 30 & $35.3 \%$ \\
\hline Female & 55 & $64.7 \%$ \\
\hline Total & 85 & $100 \%$ \\
\hline
\end{tabular}

Table-2: showed frequency and percent of malaria patients according to degree of parasitaemia

\begin{tabular}{|l|l|l|}
\hline Parasite density/+ & Frequency & Percent \\
\hline+ & 65 & $76.5 \%$ \\
\hline++ & 20 & $23.5 \%$ \\
\hline+++ & 0 & $0.0 \%$ \\
\hline Total & 85 & $100 \%$ \\
\hline
\end{tabular}

Table-3: showed the comparison between the case and control in PT and APTT

\begin{tabular}{|l|l|l|l|l|}
\hline & N & Mean & Std. deviation & Sig \\
\hline \multirow{2}{*}{ PT } & Case & 85 & 14.201 & \\
\cline { 2 - 4 } & Control & 85 & 13.668 & 0.000 \\
\hline \multirow{2}{*}{ APTT } & Case & 85 & 33.192 & \\
\cline { 2 - 4 } & Control & 85 & 33.75 & 0.735 \\
\hline
\end{tabular}

Table-4: The table was showed the comparison between the case in PT and APTT according to gender

\begin{tabular}{|l|l|l|l|l|l|}
\hline & Gender & $\mathbf{N}$ & Mean & Std. deviation & Sig \\
\hline \multirow{2}{*}{ PT } & Males & $\mathbf{3 0}$ & $\mathbf{1 4 . 4 8 7}$ & $\mathbf{1 . 1 3 0 1}$ & $\mathbf{0 . 0 6 1}$ \\
\cline { 2 - 6 } & Females & $\mathbf{5 5}$ & $\mathbf{1 4 . 4 5}$ & $\mathbf{0 . 9 6 2 2}$ & \\
\hline \multirow{2}{*}{ APTT } & Males & $\mathbf{3 0}$ & $\mathbf{3 3 . 4 7 3}$ & $\mathbf{3 . 3 3 7 9}$ & $\mathbf{0 . 5 2 3}$ \\
\cline { 2 - 5 } & Females & 55 & 33.038 & 2,7802 & \\
\hline
\end{tabular}


Table-5: The table was showed the correlation coefficient in the case according to parasitaemia

\begin{tabular}{|c|c|c|}
\hline \multicolumn{3}{|c|}{ Parasitaemia } \\
\hline Age & Correlation Coefficient & -0.046 \\
\cline { 2 - 3 }$(\mathbf{N}=\mathbf{8 5})$ & Sig. (2-tailed) & 0.673 \\
\hline PT & Correlation Coefficient & 0.058 \\
\cline { 2 - 3 }$(\mathbf{N}=\mathbf{8 5})$ & Sig. (2-tailed) & 0.596 \\
\hline APTT & Correlation Coefficient & 0.006 \\
\cline { 2 - 3 }$(\mathbf{N}=\mathbf{8 5})$ & Sig. (2-tailed) & 0.959 \\
\hline
\end{tabular}

\section{CONCLUSION}

The study concluded that the mean of PT was significantly high in case than in control while there was no differences in the mean of APTT between case and control. And there were no differences between the PT and APTT of case and control according to gender. And there is no correlation between degree of parasitemia in PT, APTT and age.

\section{RECOMMENDATION}

- The patient with malaria parasite should avoid any drug that affects hemostatic state.

- Total care must be taken in treatment of patient with inherited hemostatic disorder.

- Further studies should be done using large sample size to generalize the result.

\section{REFERENCES}

1. Francischetti, I. M., Seydel, K. B., \& Monteiro, R. Q. (2008). Blood coagulation, inflammation, and malaria. Microcirculation, 15(2), 81-107.

2. World Health Organization. (2000). Severe falciparum malaria. Trans R Soc Trop Med Hyg, 94: S1-S90.

3. Schofield, L., \& Hackett, F. (1993). Signal transduction in host cells by a glycosylphosphatidylinositol toxin of malaria parasites. Journal of Experimental Medicine, 177(1), 145-153.

4. Clark, I. A., \& Cowden, W. B. (2003). The pathophysiology of falciparum malaria. Pharmacology \& therapeutics, 99(2), 221260.

5. Perkins, D. J., Were, T., Davenport, G. C., Kempaiah, P., Hittner, J. B., \& Ong'echa, J. M. (2011). Severe malarial anemia: innate immunity and pathogenesis. International journal of biological sciences, 7(9), 1427.

6. Ansari, S., Khoharo, H. K., Abro, A., Akhund, I. A., \& Qureshi, F. (2009). Thrombocytopenia in Plasmodium falciparum malaria. J Ayub Med Coll Abbottabad, 21(2), 145-147. 